\title{
LE CRISI FINANZIARIE DALLA TULIPANOMANIA ALLA BOLLA DEL 1987: LEZIONI DALLA STORIA
}

\author{
Nota del s.c. GIUSEPPE DE LUCA (*)
}

(Adunanza del 29 novembre 2012)

\begin{abstract}
SunTo. - Nella storia delle principali crisi finanziarie dell'età moderna e contemporanea il comportamento non razionale dei suoi vari attori, dagli investitori agli speculatori per giungere fino a quello tenuto dalle stesse istituzioni, ha svolto un ruolo fondamentale. Insieme alla psicologia, alle neuroscienze e ad altre branche disciplinari, la storia economica aiuta quindi a discutere le fondamenta dell'assunzione di razionalità (che è alla base dell'edificio concettuale del mainstream economico), mostrando come questo principio è stato, ed è, più un apriori che un'effettiva realtà storica. La ricostruzione di alcune delle più importanti crisi finanziarie dell'epoca moderna e contemporanea, con particolare riguardo al comportamento irrazionale tenuto dagli attori, ne fornisce una delle motivazioni sicuramente più probanti. $\mathrm{Nel}$ modello di interpretazione più condiviso, l'euforia per il nuovo oggetto della speculazione finanziaria (dai tulipani nel 1637 alle azioni ferroviarie nel 1847, dai titoli delle compagnie commerciali nel 1720 ai fondi d'investimento nel 1987) si trasforma presto in manì, intesa come eccesso speculativo diffuso tra il pubblico, grazie ad una serie di euristiche e di altri costrutti psicologici (dal pensiero magico al comportamento gregario passando per l'euristica della rappresentatività) che concorrono a gonfiare la bolla speculativa; questa esplode appena le aspettative di guadagno oscillano e la dissonanza cognitiva scompare.
\end{abstract}

$* * *$

ABSTRACT. - Human behavior played a crucial role in the evolution of financial crises

(") DEMM - Università degli Studi di Milano, Milano, Italy.

E-mail: giuseppe.deluca@unimi.it 
over modern and contemporary age. This chapter sets out to show how irrational action - from mania to panic, from euphoria to mass hysteria - represents a key factor in the bubbles from the tulipmania of 1637 to the 1987 stock market crash. From the point of view of what really happened during the crises, investors' rationality appears an a priori belief about the way the world should work rather than a true description of the way the world has actually worked. Indeed, the prevailing model of financial crises entails the boom and the ensuing bust and is based on the episodic nature of the manias and the subsequent crises. Hence, the lesson is that even if we can't prevent someone to make a foolish of himself, the historical understanding of financial crises teaches as that it is a possible to avoid that speculative and fraudolent financial businesses ruin the less aware investors.

La centralità del comportamento umano nella dinamica delle crisi finanziarie ci è testimoniata fin dal loro primo apparire, o meglio fin da quando si è incominciato a registrarle. Già Polibio poneva la speculazione e l'avidità tra le cause della fluttuazione dei prestiti verificatasi durante gli ultimi anni della Repubblica. Allo stesso modo, il collasso creditizio che colpì Roma nell' 86 a.c. venne direttamente collegato al panico diffusosi in città a seguito della perdita di alcune immense fortune investite in Asia. I temi dell'affabulazione, dell'atteggiamento lucrativo e dell'azzardo ritornano con frequenza negli episodi di disordine finanziario che punteggiano la storia dell'Occidente dall'Alto Medioevo alla prima Età Moderna; basti ricordare a questo proposito la sconsiderata corsa ai titoli del debito pubblico veneziano a metà del XIV secolo o la precipitosa reazione degli investitori alle sospensioni dei pagamenti decretate dalla Spagna nel 1575 e nel 1596.

Certo, per questi casi non è possibile parlare di vere e proprie crisi finanziarie, tanto meno le scarne notizie sull'azione di questi operatori permettono una comprensione molto approfondita del loro ruolo nella meccanica degli spasmi. Ma le indicazioni che se ne traggono sulla 'non razionalità' degli attori e sull'importanza di questa caratteristica nell'origine delle crisi sono già estremamente significative; ben prima del famoso libro del giornalista scozzese, amico di Dickens, Charles Mackay, su La pazzia delle folle ovvero le grandi illusioni collettive, pubblicato nel 1841 (Mackay, 2000), i contemporanei erano ben consapevoli che gli eccessi speculativi erano in gran parte causati dalle tendenze momentanee della società ad essere vittima di allucinazioni di massa.

Comportamenti irrazionali, che però la teoria economica prevalente, nonostante alcune notevoli aperture, stenta ancora a riconoscere. Nel mondo dei mercati efficienti - dove il prezzo delle azioni riflette 
valori reali, e dove l'uomo razionale sa sempre ciò che vuole, ottimizzando il proprio utile e il proprio bene - non c'è spazio per gli istinti collettivi, per le pulsioni elementari, per le emozioni e per le bolle speculative irrazionali. Insieme alla psicologia, alle neuroscienze e ad altre branche disciplinari, la storia economica può aiutare a scuotere le fondamenta dell'assunzione di razionalità (che è alla base dell'edificio concettuale del mainstream economico), mostrando come questo principio è stato, ed è, più un apriori, più una fertile ipotesi, che un'effettiva realtà storica. Riducendo il rilievo delle formalizzazioni schematizzanti e dando più ascolto alla complessità del racconto umano, è tempo che l'economia accetti la realtà; e la ricostruzione di alcune delle più importanti crisi finanziarie dell'epoca moderna e contemporanea, con particolare riguardo al comportamento tenuto dagli attori, ne fornisce una delle motivazioni sicuramente più probanti.

\section{LA TULIPANOMANIA DEL 1634-37 E IL PRIMO CROLLO FINANZIARIO MODERNO}

Se intendiamo come crisi finanziaria il brusco deterioramento extraciclico della maggior parte degli indicatori monetari e creditizi (tassi d'interesse a breve termine, prezzi dei valori mobiliari, fallimenti di istituzioni finanziarie, insolvenze commerciali), la Tulipanomania, che interessò l'Olanda negli anni trenta del Seicento, non vi potrebbe essere inclusa. L'offerta di moneta e di capitali non ne risentì particolarmente; le sue conseguenze colpirono solo alcune zone del paese mentre i più danneggiati furono soprattutto gli investitori occasionali. Si trattò comunque del primo crollo di un mercato finanziario degno di questo nome, e grazie alla fortunata descrizione fattane da Mackay, divenne negli anni '50 e '60 del Novecento il prototipo stesso delle manie del mercato (Garber, 1990, p. 16).

Nei primi decenni del Seicento, la Repubblica delle Province Unite stava raggiungendo il suo massimo splendore. Nel pieno di un'interminabile guerra con la Spagna, che durò dal 1568 al 1648, l'Olanda si avviava a conquistare il primato nel grande commercio internazionale, approfittando delle incertezze delle altre potenze marittime. La Compagnia delle Indie Orientali grazie a tecniche manageriali e al monopolio commerciale realizzava profitti altissimi. Per sfuggire alla repressione cattolica, i protestanti di tutto il continente si rifugiavano 
nelle sue città e con le loro professioni, arti e cultura contribuivano a fare della piccola Repubblica lo Stato con il reddito più alto d'Europa.

La sua capitale, Amsterdam, era il perno delle principali rotte mercantili via mare, e svolgeva la funzione di magazzino del mondo. Nel 1609 vi fu aperta una Borsa merci, che nel corso di pochi anni si affermò come il principale mercato finanziario del continente, attirando capitali da tutte le plaghe e superando per volumi tutti gli altri centri finanziari esistenti; basandosi su un regolamento controllato dalle autorità, si specializzò nella contrattazione di azioni, opzioni (che erano il diritto di vendere o comprare azioni ad un prezzo stabilito durante il contratto) e di futures (contratti a termine, per ritirare o consegnare un bene ad un prezzo fisso in una certa data nel futuro), coinvolgendo ampi strati sociali; i titoli della VOC (la Compagnia delle Indie orientali) potevano infatti essere acquistati anche attraverso la partecipazione a veri e propri fondi comuni d'investimento, mentre le obbligazioni pubbliche costituivano forme d'investimento alla portata di molti risparmiatori. Si sviluppò così un effervescente mercato secondario dei titoli (cioè posteriore alla loro prima emissione e costituito dalla loro contrattazione successiva) che crebbe ad un ritmo relativamente sostenuto sino agli anni trenta del Seicento e che incoraggiò i comportamenti speculativi (Fornasari, 2006, p. 38).

La Repubblica olandese, dominata dalla borghesia mercantile, stava progressivamente perdendo una parte della propria austerità calvinista e si stava trasformando in un paese di consumatori. E nel tulipano gli agiati commercianti e professionisti trovarono l'oggetto ideale che combinava in sé il simbolo di distinzione sociale e la continua ricerca di ricchezza. Introdotti in Europa alla metà del XVI secolo dall'ambasciatore di Solimano il Magnifico, i bulbi di tulipano (tulipan in turco significa turbante) si stavano infatti diffondendo rapidamente nei giardini dei Paesi Bassi, grazie soprattutto al più grande botanico dell'epoca, l'olandese Carolus Clusius. I floricoltori delle Province Unite si appassionarono subito alla loro coltivazione, cui si prestava molto bene il ricco suolo alluvionale vicino ad Haarlem. I proprietari classificavano le sue varietà a seconda del colore dei fiori, attribuendogli titoli onorifici per sottolinearne il livello nella gerarchia dell'orticoltura. Il più ambito era il Semper Augustus con i petali color porpora imperiale, seguito da Viceré, Ammiragli e Generali, tra cui molto quotato era il General Bol.

I tulipani erano desiderati e agognati, e la scarsità fisica del prodotto finito faceva crescere i prezzi; nei primi venticinque anni del 
Seicento le varietà più esotiche toccarono prezzi favolosi: nel 1624 un Semper Augustus raggiunse la cifra di 1.200 fiorini, somma sufficiente per acquistare una modesta abitazione nella capitale. Era la stessa natura del fiore a favorire la speculazione: l'impossibilità di prevedere le sfumature di colore (che, come venne scoperto solo nel 1880, erano causate da un virus che attaccava il bulbo) aprì la strada all'azzardo; chi non poteva permettersi di investire nelle azioni delle società o del debito pubblico, poteva scommettere su un bulbo e sul tulipano che ne sarebbe venuto, di valore quindi più o meno alto; così con la crescita d'interesse si incominciò a tenere tutto l'anno il mercato, che fino ad allora si era svolto solo d'estate quando i bulbi erano sbocciati; i coltivatori marchiavano le file di bulbi e a ciascuno di essi veniva dato un numero, che corrispondeva al titolo quotato e scambiato.

Con il 1634, attirata dalle voci dei prezzi crescenti dei tulipani, che circolavano fino nel nord della Francia, arrivò sul mercato una schiera di tessitori, salumai, calzolai e contadini (che i fiorai denominarono spregiativamente 'i nuovi dilettanti'), che fece impennare i prezzi e che cambiò la natura stessa del mercato. I ricchi borghesi e i collezionisti per lo più se ne allontanarono, mentre le contrattazioni private tra individui furono sostituite da incontri informali tenuti, nelle sale delle locande, tra commercianti e speculatori. Durante la fase più intensa della manìa, tra la fine del 1636 e l'inizio del 1637, nessuna consegna di tulipani ebbe luogo: si trattava di un vero e proprio mercato di futures sui fiori, in cui i venditori promettevano di consegnare un determinato bulbo nella primavera successiva e i compratori ottenevano il diritto alla consegna, potendo regolare in contanti il contratto a termine ad ogni fluttuazione del prezzo. «Si sognavano tutti i lussi possibili. Nessuno poneva limiti alle ricchezze che avrebbe ottenuto» (citato in Chancellor, 2000, p. 27). Quelli che ottenevano profitti o che speravano di incamerarne spendevano il denaro in carrozze e cavalli. Al culmine di questa follia speculativa un Semper Augustus, che deteneva il primato assoluto delle quotazioni, raggiunse anche i 6.000 fiorini. Altri bulbi di qualità inferiore vennero ceduti a 2.500 fiorini, cifra con la quale era possibile acquistare 27 tonnellate di grano, 50 di segale, 3 di formaggio, 4 buoi, 8 maiali , 12 grosse pecore, un letto con le lenzuola e tanto altro ancora.

Nel febbraio del 1637, di fronte al propagarsi della voce che non c'erano più compratori a prezzi crescenti, il mercato crollò improvvisamente in tutto il paese; la bolla era scoppiata, i prezzi non potevano 
continuare a salire all'infinito $;^{1} \mathrm{e}$ in pochi giorni divenne impossibile vendere tulipani, a qualunque cifra. I contratti non furono saldati e i fallimenti si susseguirono. Coloro che avevano ipotecato i propri beni e scambiato i propri averi contro la speranza di un rapido guadagno patirono una perdita irrimediabile; il paesaggista Jan van Goyen, che aveva comprato una partita di bulbi per 9.000 fiorini, oltre che cedendo due suoi dipinti, morì insolvente. La giustizia olandese fu quasi ingolfata dalle denunce e dalle liti che ne scaturirono (Chancellor, 2000; Galimberti, 2008).

I maggiori banchieri, dai quali dipendeva l'economia del paese, non ne vennero però toccati e solo la parte occidentale della Repubblica ne sentì qualche riflesso. La floricoltura non venne compromessa e anche grazie a questa corsa, è oggi una delle industrie più floride del paese. A seguito della tulipanomania vennero poi sviluppate nuove tecniche di copertura dei rischi connessi allo svolgimento delle attività finanziarie; alla Borsa di Amsterdam si poterono così trattare azioni semplicemente accendendo un deposito parziale, oppure acquistando opzioni di vendita o di acquisto (put and call); attraverso queste ultime (opsie) i venditori e i compratori potevano scegliere se soddisfare il contratto oppure rescinderlo, in questo caso mediante il pagamento di un premium; vennero anche introdotti contratti a termine che potevano essere risolti trimestralmente, mediante compensazioni oppure con pagamenti in contanti dei saldi (Fornasari, 2006, p. 39).

Ma l'adozione di queste innovazioni, che consentirono anche un aumento della liquidità e una contrazione dei costi di transazione, non attenuarono affatto i comportamenti irrazionali che continuarono a caratterizzare quel mercato, così come quello degli altri paesi, anche dopo la crisi dei tulipani. L'ebreo portoghese, Joseph Penso de la Vega, che riparò in Olanda per sfuggire alla persecuzione religiosa in atto nel suo paese, ce lo dipinge, nel 1688, come una sorta di manicomio, animato da strane superstizioni e da pratiche incomprensibili. Il suo volu-

1 Secondo alcuni economisti è possibile interpretare lo svolgimento di questa crisi come uno Hog Cycle: quando i maiali sono scarsi, vengono importati, le scrofe producono molti maialini e dopo poco tempo ogni nuova femmina fa lo stesso; così la scarsità che aveva fatto impennare i prezzi, diventa abbondanza con il conseguente crollo dei valori. Allo stesso modo le nuove coltivazioni di bulbi iniziate prima dell'acme della crisi diffusero il timore che i fiori piantati anni prima sarebbero a breve stati gettati sul mercato, provocando la caduta dei loro prezzi da temuta abbondanza (Galimberti, 2002, p. 61). 
metto, intitolato significativamente Confusion de Confusiones e pubblicato ad Amsterdam in forma di dialogo tra un mercante, un filosofo e un azionista, è al tempo stesso la prima descrizione disponibile dell'attività di un mercato finanziario e la prima testimonianza analitica della sua psicologia speculativa. «Il gioco della speculazione è una cosa per folli». Ai Liefhebberen (i rialzisti) che non avevano paura di nulla contrappone di Controminatori (ribassisti), che erano completamente dominati dalla paura, trepidazione e nervosismo; c'è chi, per invocare la fortuna, mostra un atteggiamento ossessivo, «si morde le unghie, fa scrocchiare le dita, chiude gli occhi, muove quattro passi e quattro volte parla da solo, si porta la mano alla guancia come se avesse mal di denti»; c'è chi ha un comportamento maniaco-depressivo con cambiamenti d'umore violenti e incontrollati; c'è chi invece sembra sdoppiarsi, «avere due corpi, in modo che l'osservatore stupefatto vede un essere umano che lotta con se stesso». Il mercato azionario che ci tratteggia è tutt'altro che la sede del razionale bilanciamento dei valori; gli operatori sono «instabili, insani, presuntuosi e pazzi. Vendono senza sapere perché; comprano senza ragione». «Altra abilità non può esservi che quella di chiudere gli occhi e fare la prima cosa che viene alla mente, perché diverso tempo fa si è già verificato il caso che, nel pieno furore della guerra, le azioni siano salite fino alle nubi». Al tempo stesso è un luogo che emana un'attrazione irresistibile, e chi è entrato una volta «nel cerchio magico della Borsa è in preda di un'eterna agitazione e si trova in una prigione le chiavi della quale sono nel fondo dell'Oceano e le cui sbarre non si aprono mai» (de la Vega, 1688).

\section{L'implosione del Sistema di LAW E LA Bolla Dei Mari DEL SUD DEL 1720 ALL'ORIGINE DEL PRIMO CRASH FINANZIARIO INTERNAZIONALE}

Agli ingredienti che agirono nella crisi dei tulipani, si aggiunsero nelle due bolle parallele del 1719-20, di Parigi e di Londra - tutti quegli elementi che derivavano dalla progressiva maturazione degli stessi sistemi finanziari, come la moneta fiduciaria (sottoforma di banconote), gli istituti di emissione, la gestione e la circolazione internazionale dei titoli, con l'effetto di aggravarne e di propagarne notevolmente gli effetti.

Il Sistema di John Law si sviluppa nella drammatica situazione finanziaria in cui la Francia si dibatteva dopo la conclusione della 
Guerra di successione spagnola nel 1713. Le urgenze belliche di Luigi XIV avevano gonfiato il debito pubblico fino a quasi 3 milioni di lire tornesi, ripercuotendosi negativamente sia sulla stabilità monetaria che sulla disponibilità di capitali. Alla sua morte, nel settembre del 1715, il paese non era più in grado di pagare gli interessi in scadenza e fu in questa situazione, prossima al collasso e aperta ad ogni suggerimento, che trovarono ascolto e realizzazione le teorie e le proposte del finanziere scozzese.

Nato ad Edimburgo nel 1671, John Law aveva acquisito precocemente una profonda conoscenza del sistema bancario scozzese, lavorando nella ditta del padre, un affermato operatore del settore. Nel 1688, si era trasferito a Londra dove, mentre dissipava la sua cospicua eredità, venne istruito negli affari monetari da Thomas Neale, direttore della Zecca e consigliere segreto della Banca d'Inghilterra, che, fondata nel 1694 stava proprio allora avviando la prima forma di circolazione monetaria cartacea nazionale. Venne poi in contatto direttamente con le pratiche finanziarie continentali quando, nel 1697, dovette rifugiarsi ad Amsterdam, in seguito all'uccisione in duello di un rivale in amore; lì si mantenne con speculazioni sui titoli del debito pubblico e con il gioco delle carte, sfruttando abilmente le sue doti matematiche nel calcolare le probabilità. Durante diversi viaggi all'interno della stessa Olanda, in Italia e in Francia, Law si dedicò allo studio del funzionamento dei mercati finanziari e delle principali istituzioni bancarie dell'epoca, rimanendo impressionato dai benefici potenziali che l'introduzione della valuta cartacea aveva prodotto in Inghilterra e nei Paesi Bassi, rispetto alle nazioni che non l'avevano realizzata. Fra il 1701 e il 1704 preparò alcuni progetti per l'istituzione di una banca che emettesse biglietti garantiti non da metalli preziosi ma da beni fondiari. Il nucleo teorico delle sue proposte stava nella convinzione che il volume del circolante condizionasse le attività commerciali e con esse anche il benessere generale di un paese; ispirandosi alla circolazione sanguigna diffusa da William Harvey nel terzo decennio del Seicento, Law sosteneva che come «quando il sangue non circola attraverso il corpo, il corpo langue così quando non circola il denaro, la vita economica dello Stato ristagna» (citato in Murphy, 1997); a suo avviso, invece, come argomentava in Money and Trade Considered with a Proposal for Supplying the Nation with Money (Edimburgo, 1705), grazie alla moltiplicazione del circolante, il denaro sarebbe diventato più a buon mercato, gli interessi del credito si sarebbero abbassati, gli investimenti 
sarebbero cresciuti e un maggior numero di popolazione sarebbe stata coinvolta nel processo produttivo. Ma la moneta metallica era scarsa e, a causa delle oscillazioni nell'offerta di oro e di argento, sottoposta a continue tensioni inflattive. Al contrario, secondo Law, la moneta cartacea sarebbe stata il mezzo più adatto a moltiplicare la quantità di circolante dato che, oltre ad essere di costo quasi nullo, la sua emissione avrebbe potuto essere facilmente regolata in relazione alle esigenze del commercio e della produzione.

Rifiutate dai governi della Scozia, dell'Olanda e del Ducato di Savoia, come abbiamo già anticipato, le sue tesi trovarono invece accoglienza presso Filippo d'Orléans, reggente della corona francese, afflitto da una situazione finanziaria disperata a causa dell'enorme debito pubblico, della cronica scarsità di moneta in circolazione e della grave carenza di investimenti. Il piano dello scozzese, che prometteva di ridurre il debito pubblico e di stimolare il commercio e la manifattura francese, persuase la reggenza a concedergli, nel maggio del 1716, l'autorizzazione ad aprire a Parigi una banca privata sotto forma di società anonima (per azioni).

Aveva così inizio la costruzione del Sistema di Law, destinato a svilupparsi, con cadenza quasi annuale. Con sorpresa dello stesso governo, la Banque Générale ebbe subito un notevole successo; accettò depositi e scontò cambiali dapprima al $6 \%$ e poi al $4 \%$ (contro il $30 \%$ preteso dagli operatori privati); dietro versamento di moneta metallica emise, già nel primo semestre di vita, biglietti pagabili a vista per 61 milioni di lire tornesi e dato che il loro valore veniva espresso in scudi di banca, vale a dire in termini inalterabili, essi anche se non avevano corso legale furono accettati con favore viste le oltre quaranta svalutazioni delle monete d'oro e d'argento subite nei venticinque anni passati; inoltre un decreto ne impose l'accettazione per il pagamento delle imposte. L'istituto distribuì anche buoni dividendi e Law ne ottenne subito stima e prestigio. Nel 1717, grazie alla sua crescente credibilità e al sotteso intervento sul ripianamento del debito pubblico, lo scozzese ottenne l'autorizzazione per costituire la Compagnie d'Occident (comunemente nota come la Compagnie du Mississipi); in cambio del monopolio del commercio e dei diritti di sfruttamento della colonia francese della Luisiana per venticinque anni, il suo capitale sarebbe stato costituito da titoli di Stato che i proprietari avrebbero potuto scambiare con azioni della compagnia (secondo la pratica dell' 'innesto', inaugurata in Inghilterra e oggi nota come debt/equity swap). Di fronte 
alle promesse ricchezze del Mississippi, astutamente propagandate da Law, obbligazioni governative per 100 milioni furono convertite facendo rivalutare anche quelle rimaste sul mercato.

L'anno successivo, oramai da una posizione di forza all'interno della finanza francese, John Law trasformò la Banque Générale in Banque Royale, attuando così la sua idea originaria di un istituto pubblico che avrebbe emesso banconote non dietro depositi dei privati ma su garanzia dello Stato e secondo i bisogni dell'economia; furono così stampati 950 milioni di biglietti non più in scudi di banca ma in moneta corrente, le lire tornesi, quindi suscettibili di subire variazioni. La circolazione cartacea si diffuse anche in provincia e diverse filiali furono aperte a Lione, La Rochelle, Tours, Amiens e Orléans. Nel maggio del 1719 la Compagnie du Mississipi, che aveva già esteso i suoi interessi all'Africa e ottenuto la privativa della vendita del tabacco, assorbì anche quelle che negoziavano con l'Asia e mutò denominazione in Compagnie des Indes, assumendo di fatto il monopolio del commercio estero francese; nei mesi successivi si aggiudicò anche l'appalto della riscossione delle tasse indirette e il diritto esclusivo di coniare monete. A ottobre Law, da una posizione dominante nella vita economica nazionale, mise in atto il piano per consolidare il debito pubblico, accettando i titoli di Stato al valore nominale in pagamento del prezzo di un'azione della Compagnia; nessun detentore di obbligazioni pubbliche fu forzato a scambiarle ma dato che queste valevano sul mercato libero meno della metà del loro valore ufficiale, molti erano desiderosi di disfarsene per comprare quote della Compagnie per le quali venivano accettate alla pari. Inoltre le nuove azioni (o la differenza rispetto allo scambio) potevano essere pagate in rate mensili e questo le rese alla portata anche dei piccoli investitori. Come lo scozzese aveva auspicato, la speranza in un dividendo (pubblicizzato in modo strategico) e la corsa all'acquisto dei titoli della Compagnie ne fecero aumentare rapidamente il loro prezzo di mercato: il valore di un'azione da 500 lire salì, nel gennaio del 1720, a 18.000 con una rivalutazione del $3600 \%$.

La domanda assunse dimensioni che neppure lo stesso Law aveva previsto, scatenando una speculazione febbrile. A Parigi, in Rue Quincampoix, una stretta stradina tra rue St Denis e rue St Martin, dove la società aveva il suo ufficio di emissione delle azioni e dove avvenivano le contrattazioni, affluivano persone non solo dalla Francia ma anche da altri paesi, con l'attrattiva di un repentino arricchimento. Un funzionario dell'ambasciata britannica descriveva la 
strada «affollata dall'alba a notte fonda di principi e principesse, duchi e duchesse eccetera, in una parola tutti i grandi di Francia. Vendono proprietà e impegnano gioielli per acquistare Mississippi» (citato in Murphy, 1997, pp. 201-2). Un lustrascarpe della Savoia guadagnò 40 milioni, un servitore 50 e un esponente dell'aristocrazia, come il duca di Borbone, 60. Fu in questo periodo che venne coniata la parola milionario, proprio per i mississipiens, che gareggiavano con la nobiltà in carrozze e lussuose dimore.

Come scrive il suo connazionale Mackay, Law era idolatrato dal popolo volubile: «ogni volta che usciva, tanto grande era la folla che seguiva la sua carrozza che il reggente gli mandò una squadra di soldati a cavallo come scorta permanente per sgombrargli la strada» (Mackay, 2000 , p. 32). A più riprese vennero emesse azioni della Compagnie fino ad un valore nominale complessivo di 312 milioni. «Lillusione era sostenuta dalla straordinaria avidità della gente e, più alto diventava il prezzo delle azioni della Compagnia delle Indie e del Missisipi, più billets de banque venivano emessi per tenere il ritmo» (Mackay, 2000, pp. 33-34). Il debito pubblico si ridusse così quasi completamente, e il costo del denaro si abbassò in modo radicale, migliorando le condizioni del credito.

Bastò però che, nel gennaio del 1720, la Compagnie des Indes annunciasse un dividendo di entità irrisoria (il $2 \%$, che era invece elevato se riferito, non al valore di mercato, ma a quello nominale) per avviare il ribasso delle azioni; alcuni cominciarono a realizzare, vendendo i titoli e cambiando le banconote - la Banque Royale ne aveva emesse per ben 1.200 milioni di lire tornesi per sostenere l'acquisto delle azioni! - in moneta metallica; contemporaneamente gli investitori stranieri spostarono i propri mezzi sul mercato rialzista di Londra e di Amsterdam. Law, nominato nel frattempo duca dell'Arkansas e controllore generale delle Finanze, fece dichiarare l'inconvertibilità dei biglietti e procedette alla fusione fra la Banque e la Compagnie, completando così l'architettura istituzionale del suo sistema; ma tutto questo non poté evitare la diffusione del panico, e né la chiusura temporanea della Borsa né il sostegno diretto dello scozzese riuscirono a frenare la discesa delle azioni. In luglio la banca dovette sospendere i pagamenti e il suo sistema implose come un castello di carta (Murphy, 1997; Gleeson, 2000); Law fu costretto a fuggire, mentre la satira incominciò a bersagliarlo insieme alla pazzia collettiva che lo aveva sostenuto: una caricatura lo rappresentava seduto in un grande calderone, «riscaldato 
dalla follia popolare, circondato da un'impetuosa moltitudine intenta a versare oro e argento dentro il calderone e felice di ricevere in cambio pezzi di carta che egli distribuisce a piene mani», un'altra raffigurava la «Dea delle Azioni, sul suo carro trionfale, guidato dalla Dea della Follia ... [mentre] gli agenti di queste compagnie [del Mississippi, dell'Africa occidentale], riconoscibili dalle lunghe code di volpe e dall'aspetto astuto, girano i raggi delle ruote, dove sono segnati i nomi delle varie azioni e il loro valore, ora alto ora basso, a seconda dei giri delle ruote» (Mackay, 2000, p. 45).

Il crollo di Parigi fu amplificato a Londra dall'esplosione contemporanea della bolla della Compagnia dei Mari del Sud, le cui azioni si ridussero ad un quarto del loro valore tra il settembre e l'ottobre dello stesso anno (Neal, 1990).

Per risollevarsi dall'ingente debito pubblico, prevalentemente legato al finanziamento della guerra di successione spagnola, anche l'Inghilterra era ricorsa alla pratica dell'innesto, che del resto era stata la sua Banca d'emissione ad inaugurare nel 1697. Lo schema della Compagnia dei Mari del Sud, fondata da John Blunt nel 1711, era di convertire il debito statale nelle appetibili azioni della società, a cui due anni dopo era stato attribuito il ricco monopolio del commercio degli schiavi neri con l'America Latina; le sue quote vennero offerte in quattro tranches, permettendo la rateazione del pagamento, concedendo prestiti con azioni a garanzia e distribuendo ingenti dividendi. La conversione del debito avrebbe avuto successo solo se il prezzo delle azioni fosse continuato a salire, e tutti i suoi sostenitori si adoperarono per questo attraverso una campagna ben orchestrata di annunci; per sostenere una massiccia operazione di lobbying, un buon numero di parlamentari, di cortigiani, di favorite del re, di altissimi funzionari avevano ricevuto quelle che oggi si chiamerebbero stock options, cioè il diritto di comperare azioni a un certo prezzo, per poi rivenderle quando il prezzo fosse stato più alto. L'euforia cedette così subito il passo alla manìa: secondo il famoso poeta Alexander Pope era «ignominioso non partecipare» alla sottoscrizione delle azioni. La febbre della speculazione contagiò anche le donne, che incominciarono ed essere assidue frequentatrici di Exchange Alley, sede degli scambi (Chancellor, 2000; Galimberti, 2008, pp. 141-2). Nacquero ovunque innumerevoli compagnie (190 nel 1720), che «vennero presto chiamate col nome di bolle» e che avevano gli scopi più balzani e improbabili ma le cui azioni erano subito esaurite (Mackay, 2000, p. 63). 
Quando nel marzo arrivò da Parigi la notizia del crollo della Compagnie des Indies, il prezzo delle azioni della South Sea Company ebbe un ulteriore rialzo. Isaac Newton, che era stato direttore della Zecca, interrogato sull'andamento di quelle azioni rispose: «posso calcolare il moto dei corpi celesti ma non la pazzia della gente», e coerentemente vendette quelle che possedeva guadagnando 7.000 sterline, ma rientrò nel mercato poco prima del crollo e finì per perderne 20.000 (citato in Galimberti, 2008, p. 145). Oramai la bolla aveva acquistato vita propria, la leva finanziaria, implicita nella possibilità di acquistare a rate, aveva messo in moto il meccanismo: con una piccola somma si potevano fare fortune. Il prezzo andava su oggi perché era andato su ieri, e l'eccesso speculativo aveva progressivamente investito anche le classi non agiate e compratori provenenti da Amsterdam, Lisbona e dal Brasile. Ma la promulgazione, il 9 giugno del 1720, della legge passata alla storia con il nome di Bubble Act, finalizzata a limitare il collocamento al pubblico delle azioni di società di nuova costituzione e richiesta dai direttori della South Sea Company proprio per togliere di mezzo la concorrenza, finì per generare il panico nel mercato azionario e per fare precipitare le sue azioni.

Il primo crash del mercato azionario internazionale dimostrava eloquentemente, oltre ai meccanismi irrazionali nel comportamento degli attori, la fragilità e la difficoltà di una finanza che provava a misurarsi, sullo sfondo di piazze integrate, con l'invenzione della cartamoneta, delle società per azioni e con il contenimento del debito pubblico. In Francia la memoria dell'esperienza dello scozzese, morto povero a Venezia nel 1729, ritardò notevolmente la creazione di una struttura creditizia adeguata all'economia: per anni non si osò neppure pronunciare il vituperato nome di 'banca'. Dopo il crollo del Sistema di Law - dovuto più al tentativo di convertire il debito pubblico in azioni societarie che non alle sue concezioni monetarie (che si meriteranno l'apprezzamento di Joseph Schumpeter e che prefigurano l'attuale standard monetario, non più basato sulla merce ma sulla convenzione) - il paese transalpino espresse filosofi e pensatori come Quesnay e Mirabeau, che invocavano politiche protezionistiche e affermavano che il commercio, le società per azioni e le speculazioni di mercato erano contrarie agli interessi dello Stato (Fornasari, 2006, pp. 45-47). In Inghilterra, invece, la classe politica e gli operatori economici furono in grado di trarre opportune indicazioni dal crollo e gli effetti della crisi del 1720 non furono sistemici, a parte i vincoli posti 
al collocamento pubblico delle azioni e all'operatività delle compagnie dal Bubble Act: la South Sea company continuò ad esistere e gli investitori stranieri continuarono ad avere fiducia nelle attività finanziarie inglesi.

\section{MANİE NELL'ETÀ DELL'INDUSTRIA E DELLA COMUNICAZIONE: IL CRACK FERROVIARIO DEL 1847}

Dalla metà del XVIII secolo, anche grazie al buon contenimento degli effetti della crisi del 1720, l'Inghilterra si stava avviando, per prima, sulla strada di quel processo che avrebbe definitivamente segnato il tramonto dell'antico regime e l'inizio della nuova era industriale; il cambiamento sarebbe stato radicale e una nuova Weltanschaunng economica si sarebbe affermata grazie anche ad una sequela straordinaria di innovazioni, tra cui la ferrovia era una delle più rilevanti. Fino agli anni venti dell'Ottocento, la costruzione di strade ferrate era però rimasta circoscritta ad ambiti locali e funzionanti per lo più a trazione animale; l'innovazione decisiva fu costituita dall'applicazione della forza vapore e dalla conseguente diffusione della locomotiva; da quel momento la costruzione di una rete ferroviaria nazionale progredì attraverso una seria di improvvise accelerazioni seguite da altrettanti bruschi arresti: tra il 1824 e il 1829 vennero costruite più di 70 miglia di strade ferrate e tra il 1831 e il 1837 oltre 500 (Fornasari, 2006, pp. 55-7).

Alla fine del 1844 la situazione era favorevole per un nuovo balzo: i tassi d'interesse erano ad un livello bassissimo e il grano, molto abbondante, costava poco; i costi delle costruzioni ferroviarie erano scesi e i profitti delle società crescevano rapidamente. Nel gennaio del 1845 vennero così avviati sedici nuovi progetti ferroviari ed entro qualche mese furono registrate oltre cinquanta nuove compagnie. Le pubblicità che invitavano a sottoscrivere le azioni delle società costruttrici inondarono i giornali; le nuove compagnie erano osannate dagli amici nella stampa specializzata e le loro azioni erano spinte verso l'alto dagli operatori del mercato azionario. Le invenzioni e le novità hanno sempre eccitato gli speculatori. Ferrovie di dubbio valore erano reclamizzate in modo acritico negli editoriali, mentre $\mathrm{i}$ giornali ricevevano così una parte delle centinaia di migliaia di sterline spese ogni settimana per pubblicizzare questi prospetti. Il Times scriveva, nell'ottobre del 1845 , che «la manìa della pubblicità è stata tale che si sono lanciati giornali 
che non avevano nessuna intenzione di essere venduti o di essere letti ma soltanto di intercettare una parte del denaro che veniva quotidianamente dilapidato in annunci preliminari e prospetti». La creazione più biasimevole della manìa ferroviaria fu «la speculazione della stampa» (citato in Chancellor, 2000, p. 128).

Le iniziative ferroviarie, il cui successo e il cui valore erano amplificati in questo modo dai giornali, avevano iniziato ad attirare i risparmi di un numero crescente di persone appartenenti a tutti i ceti sociali, non sempre capaci di valutare adeguatamente le prospettive di sviluppo dei diversi progetti. «Un capitale cieco, alla ricerca del suo 5\%, una cosa completamente diversa dal capitale perspicace degli uomini d'affari quaccheri delle Midlands e del Nord, si era accumulato per i profittatori» (citato in Fornasari, 2006, p. 58). La manìa fu particolarmente intensa nella provincia: «da Edimburgo a Inverness tutti sono impazziti per la ferrovia. Il paese è un manicomio di lunatici ferroviari», scriveva nel suo diario il poeta Wordsworth (citato in Chancellor, 2000, p. 129) La speculazione fu facilitata anche dalla diffusione di banche d'investimento che erogavano prestiti in cambio di garanzie sulle azioni ferroviarie, e nuove borse furono fondate al Nord per facilitare il loro traffico.

Durante i primi sei mesi del 1845 , tutti i nuovi piani ferroviari distribuirono profitti, anche quelli previsti per le zone più impervie e disabitate, le cui richieste erano state respinte dal Parlamento. L'irrazionalità della situazione era denunciata anche da "The Economist": «il valore di mercato non dipende dall'idea che ci si fa della riuscita finale dell'impresa, ma piuttosto da quanto a lungo le circostanze tenderanno a sostenere o aumentare la fame del pubblico per la speculazione» (citato in Chancellor, 2000, p. 131). La corruzione e la frode si aggiunsero a questi eccessi lucrativi e nell'ottobre del 1847, quando la Banca d'Inghilterra annunciò che non avrebbe più concesso anticipi sui titoli a causa del basso livello delle sue riserve, il tracollo della Borsa e delle azioni ferroviarie fu inevitabile. Qualche anno dopo venne scritto che «nessun altro panico fu mai così disastroso per le classi medie. Raggiunse tutti i focolari, rattristò i cuori del paese. Intere famiglie furono rovinate. Non c'era quasi città in Inghilterra in cui non si fosse registrato un disgraziato suicidio ... tutti i legami sociali si dissolsero» (citato in Chancellor, 2002, p. 137).

Di recente è stato osservato come il parallelo più vicino a questa manìa sia stato rappresentato dallo sviluppo di Internet intorno al 1995; le analogie più stringenti riguarderebbero l'uso di un linguaggio simile 
nel descrivere le trasformazioni indotte dalle due innovazioni, l'emergere di una pubblicistica specializzata in entrambi i casi, e l'ampiezza della febbre speculativa che, in tutte e due le circostanze, spinse alla creazione di nuovi e più ampi mercati azionari. A seguito del crollo del 1847, l'Inghilterra adottò delle innovazioni tecnologiche ed istituzionali finalizzate a ridurre le asimmetrie informative palesatesi durante la crisi, in particolare migliorando la legislazione commerciale e societaria, la corporate governance delle imprese e i mezzi informativi a disposizione del pubblico; elementi che avrebbero contribuito al pieno dispiegarsi del capitalismo liberale tra la fine del secolo e la vigilia della Prima Guerra Mondiale.

\section{LA CRISI DEL 1929 E LA FINE DI UNA NUOVA ERA}

La crisi del 1929, e la Grande depressione che ne seguì, segnarono un punto di svolta epocale nella storia e nella teoria economica del mondo occidentale, mostrando tutti i limiti delle ortodossie e degli automatismi che avevano governato il sistema economico fino allo scoppio del primo conflitto mondiale. Colpì tutte le maggiori economie produttrici di beni manufatti, così come di materie prime, ed ebbe una scala mondiale, anche se con due focolai principali, uno nelle Americhe, gli Stati Uniti, e uno nel vecchio continente, la Germania.

La crisi finanziaria si combinò con una crisi di sovrapproduzione, che aveva le sue radici nella dislocazione dell'economia globale prodottasi in seguito al conflitto; durante la Grande Guerra, la capacità produttiva agricola ed industriale extraeuropea era cresciuta e quando anche l'Europa, dopo il conflitto, riprese a produrre, si manifestò un potenziale produttivo in eccesso che trascinò verso il basso i prezzi dei beni primari ben in anticipo rispetto al 1929 .

In questa situazione, per i paesi che avevano contratto debiti di guerra, e in particolare per la Germania, soffocata dalle richieste di riparazioni, diventava difficile procurarsi la valuta pregiata necessaria per pagare gli interessi ai creditori esteri; il rafforzamento delle organizzazioni dei lavoratori rendeva poi difficile per i datori di lavoro tagliare i salari in risposta alla diminuzione dei prezzi, e così in molti casi la contrazione dei profitti, causata dall'aumento dei salari reali, costrinse le imprese a licenziare, con l'effetto di ridurre ulteriormente la domanda di beni. Anche il commercio internazionale era sconvolto da una muta- 
ta dislocazione delle produzioni agricole, il cui accumulo di scorte, dal 1925 in poi, influì sul declino dei prezzi con ragioni di scambio peggiorate del $25 \%$, rispetto ai prodotti industriali; contro il ripristino dell'ordine monetario e finanziario prebellico pesava l'imposizione di riparazioni alla Germania. La dipendenza ideologica dal mito dell'oro giocò poi la sua parte nell'impossibilità di ristabilire, dalla metà degli anni '20, uno stabile sistema monetario internazionale che consentisse la ripresa dei movimenti di capitale; i vari paesi vi aderirono in ordine sparso e con parità di cambio non concordate su basi multilaterali, e furono presto costretti a sospendere la convertibilità delle loro valute.

Di fronte alle difficoltà di ripresa del vecchio continente, gli Stati Uniti, che dal 1913 erano la prima potenza economica e finanziaria mondiale, stavano vivendo una fase di intensa prosperità, cominciata, nel 1924, con l'elezione del presidente Calvin Coolidge; l'estensione del libero commercio, il calo dell'inflazione, i progressi nella produttività industriale e un metodo più scientifico nella gestione delle società stavano determinano un 'rinascimento industriale'; ad osservatori professionisti, come Irving Fisher, sembrava che il cielo fosse l'unico limite a questa espansione; nel 1928, durante il discorso di accettazione della candidatura per le presidenziali, Herbert Hoover dichiarò che la fine della povertà era alle porte e che una nuova era stava cominciando (Chancellor, 2000, pp. 183-7).

Un numero crescente di famiglie americane desiderava dotarsi di automobili e di altri beni di consumo durevole, e radio, frigoriferi, macchine e abiti potevano tutti essere acquistati a credito. I titoli delle imprese produttrici di questi beni registrarono degli aumenti stupefacenti: la quotazione della Rca, la società tecnologica per eccellenza degli anni '20, crebbe del 939\% tra il 1925 e il 1929. L'euforia incoraggiò la corsa all'emissione di nuove azioni e nel 1929 furono lanciati sul mercato titoli per 6 miliardi di dollari; le società di brokeraggio si ingrandirono rapidamente e proliferarono nuove istituzioni finanziarie, note come investment trust, progettate per capitalizzare sul boom del mercato borsistico. Allo stesso tempo, molti piccoli investitori si affidavano alla leva finanziaria per potenziare la propria esposizione al mercato azionario ricorrendo ai prestiti concessi dagli intermediari per comprare azioni al margine, sborsando così di tasca propria solo una frazione del prezzo di acquisto. Gli scambi arrivarono ad essere trenta volte il Pil. Nonostante le stime sui giocatori di Borsa alla fine degli anni venti oscillino tra uno e due milioni, su una popolazione americana di oltre 
120 milioni, il mercato rialzista attrasse un interesse collettivo che andava oltre questi numeri; celebrità del mondo dello spettacolo, come Groucho Marx, Charlie Chaplin e Irvin Berlin, specularono con prestiti garantiti da azioni. «Gli americani trovarono nel mercato azionario dei ruggenti anni venti una religione secolare, le qualità ludiche, il cinismo e il materialismo», che riflettono lo spirito dell'età del jazz. Il grande Gatsby di F. Scott Fritzgerald (che vide la stampa nel 1925), in cui si intrecciano speranza e disillusione, è una parabola dell'epoca (Chancellor, 2000, pp- 194-5).

A dispetto dell'emergere di molti argomenti contrari alla rivalutazione delle azioni (il credito per le vendite rateali aveva raggiunto il limite massimo poiché i salari non crescevano e gli alti tassi di interesse incominciavano ad appesantire i prestiti per l'acquisto delle azioni), la prospettiva di continui profitti derivanti dalla speculazione non veniva scalfita. Nell'introduzione all'edizione del 1932 dell'opera di Makcay, Bernard Baruch evidenziò che gli speculatori americani del 1929 mostravano i tratti della psicologia delle folle e, citando il detto di Shiller, affermò che tutti gli individui come membri di una moltitudine diventano improvvisamente degli stupidi. Edward Chancellor sostiene che, nella mentalità del mercato rialzista di allora si ritrovano alcuni elementi che Freud e lo psicologo francese del XIX secolo, Gustav Le Bon, identificarono in quella delle folle, come invincibilità, irresponsabilità, impeto, contagio, variabilità, suggestionabilità, allucinazione collettiva e inferiorità intellettuale (Chancellor, 2000, p. 202). Ignorando le cattive notizie, questi attori mantenevano un comportamento di dissonanza cognitiva perché desideravano ardentemente i rapidi profitti delle speculazioni. Invece di ragionare si esaltavano con le voci dei favolosi arricchimenti ottenuti da servitori, autisti, mandriani e quanti altri. Nell'estate del 1929, l'americano medio era caduto in una sorta di trance, da cui si risvegliò bruscamente giovedì 24 ottobre 1929, quando, mezz'ora dopo l'apertura di Wall Street, molte azioni persero dieci punti e molte altre restarono senza offerta. Senza un evidente detonatore, era scoppiato il panico; il martedì successivo il mercato azionario venne travolto da un'ulteriore ondata di ordini di vendita, poiché i prestiti garantiti da azioni costringevano gli speculatori a recuperare le proprie azioni. Alla vigilia del crash, gli americani detentori di azioni ordinarie erano dieci milioni (White, 1990).

Nei mesi che seguirono il crollo di borsa si ebbero ondate di panici bancari. I depositanti prendevano d'assalto gli sportelli delle banche 
perché temevano un loro coinvolgimento nella crisi e perché avevano realmente bisogno di fondi per rimborsare i debiti giunti a scadenza e a cui non potevano far fronte con la liquidazione rovinosa dei valori mobiliari in loro possesso. La banca centrale americana, la Federal Reserve, non fece che aggravare la situazione restringendo la quantità di credito disponibile, e spingendo così le banche a vendere i loro titoli alla ricerca spasmodica di liquidità, con l'effetto di fare precipitare il loro valore e di peggiorare la situazione del sistema creditizio. Vennero proclamate diverse vacanze bancarie per frenare la corsa agli sportelli, ma tra il 1931 e il 1933 fallirono oltre 11.000 aziende credito, nonostante le famose fireside chats del successore di Hoover per rassicurare gli americani sulla loro solidità.

Come mai in precedenza, i contemporanei furono ben consapevoli della dimensione psicologica della crisi. Nel suo discorso inaugurale, il presidente Roosevelt dichiarò: «The only thing we have to fear, is fear itself» (citato in Akerlof, Shiller, 2009, pp. 11-12). John Maynard Keynes parlò di un «fallimento nelle articolazioni immateriali della mente». $\mathrm{Ma}$ entrambi erano anche convinti che una parte della responsabilità della crisi fosse da ricondurre alla scorrettezza di alcuni comportamenti finanziari: il presidente puntò il dito contro «i trafficanti di denaro senza scrupoli» di Wall Street, mentre Keynes nella sua Teoria generale paragonò il mercato azionario ad un casinò (Galimberti, 2008).

Nel 1931, poi, il panico finanziario si scatenò anche in Europa. Tra il maggio e il luglio cadde in dissesto il Credit Anstalt, la principale banca austriaca. Il contagio si estese nel mese successivo alle banche tedesche, ma la chiusura temporanea degli sportelli, evitò il peggio per l'intero sistema. I pochi capitali esteri, in gran parte a breve termine, rimasti dopo la prima ondata di fuga dell'autunno 1930, abbandonarono senza altre esitazioni i due paesi in conseguenza dell'esito delle elezioni favorevoli al partito nazista. Le banche centrali, al di là di errori tecnici, non intervennero in maniera risolutiva con un'espansione monetaria, perché ciò avrebbe minacciato la convertibilità aurea, già compromessa dai deflussi valutari. La perdita di fiducia riguardò così tutti i paesi con bilance dei pagamenti in deficit che, ora, non potevano ottenere nuovi crediti dalle banche americane. Le banche inglesi, che vantavano crediti consistenti verso la Germania subirono ritiri di fondi a livello internazionale, che comportarono la svalutazione della sterlina e l'abbandono della convertibilità, avvenuta il 21 settembre 1931. Altri paesi seguirono il suo l'esempio, per ridare competitività alle proprie 
esportazioni e a tentare di raddrizzare i propri conti sull'estero. Anche gli Stati Uniti, nella primavera del 1933, svalutarono il dollaro del $33 \%$, ma queste manovre competitive annullavano a vicenda gli effetti desiderati per ridare slancio alle singole economie nazionali. In pochi anni il Gold Exchange Standard internazionale, faticosamente ricostruito attorno alla metà degli anni 20, era definitivamente cancellato.

La nuova potenza egemone sul piano economico e finanziario non si assunse la responsabilità di organizzare forme di cooperazione internazionale che potessero arginare la depressione prima che questa dilagasse, aggravata dagli effetti della crisi finanziaria. Sul piano interno, la depressione investì le varie categorie sociali e, specialmente, i settori più deboli della popolazione, le cui fila di sottoproletari e diseredati furono ingrossate non solo da salariati senza lavoro, contadini e proprietari terrieri medi e piccoli, ma anche da un gran numero di borghesi agiati gettati sul lastrico e sradicati di netto dalla loro posizione economica, abitudini, stili di vita, relazioni sociali e umane. La disoccupazione raggiunse punte altissime sia negli Stati Uniti che in alcuni paesi europei. Sul piano politico tutto ciò alimentò l'affermazione del nazismo, esasperò i nazionalismi economici e politici, che, a loro volta, fecero precipitare l'Europa nella seconda guerra mondiale. Le politiche utilizzate fino ad allora dettero una pessima prova in una congiuntura in cui la sincronia quasi generale privò l'economia interna e internazionale di fattori di compensazione e della possibile azione di un prestatore di ultima istanza. I meccanismi di aggiustamento spontaneo, sui quali si confidava per riportare l'economia al pieno impiego, non funzionarono e le ricette teoriche prevalenti non furono in grado di fornire risposte efficaci. Solo l'abbandono dei vincoli di bilancio e il sostegno pubblico della domanda, sulla scorta degli stimoli dell'autore della Teoria generale, furono in grado di avviare la ripresa, prefigurando il paradigma fordista-keynesiano che nell'immediato dopoguerra sarebbe stato responsabile del più intenso periodo di crescita economica del mondo occidentale.

\section{LA BOLLA DEL 1987: «CI VUOLE UNO PSICOLOGO!»}

Negli anni 70 del Novecento si era esaurito l'ordine economico, caratterizzato dalla stabilità e dall'intervento dello Stato, lungo i cui binari si era realizzato uno straordinario sviluppo. All'ortodossia key- 
nesiana si stava sostituendo progressivamente quella del monetarismo, secondo la quale i mercati sono il modo migliore per distribuire le informazioni e assicurare gli incentivi alla crescita economica, indipendentemente dalle disuguaglianze che possono sorgere. Con l'elezione della Thatcher in Inghilterra, nel 1979, e di Ronald Reagan, nel 1980, a capo degli Stati Uniti, questa ideologia si traduce in una precisa linea di politica economica, volta alla liberalizzazione del mercato, e alla flessibilità dell'iniziativa. Negli Usa si instaurò un clima complessivo di profonda fiducia e l'economia cominciò a crescere dopo la flessione del decennio precedente. Il potere dell'antitrust venne sempre più limitato e i mercati dell'energia, delle banche, delle telecomunicazioni e delle utilities vennero liberalizzati. La deregulation portò il mercato ad essere l'unico giudice di se stesso. La Fed, già a partire dagli anni Settanta, dopo la fine del sistema di Bretton Woods, stava introducendo nuovi strumenti finanziari, volti a stabilizzare i regimi di cambio, quali le opzioni su azioni, nel 1972, i future su oro, petrolio e obbligazioni, e nel 1982, le opzioni su valute. Ma fu nel 1981 che si realizzò una vera svolta, attraverso «la possibilità di regolare una transazione in moneta e non con la consegna fisica del prodotto sottostante». Le conseguenze di questa concessione furono molteplici, ma l'esito più interessante si ebbe con l'istituzione di nuovi indici azionari, quale lo Standard \& Poor 500, nel 1982.

Le novità in campo finanziario sembravano non finire mai; venne inventato lo stripping, una tecnica che consente la separazione dell'obbligazione in due parti, il capitale ed i dividendi; si giunse alla titolarizzazione, la commercializzazione, cioè, di beni in precedenza illiquidi; fecero la loro apparizione anche i Cars (Collateralize Automobile Receivables), gli Spins (titoli di debito legati all'indice S\&P), e il contratto di swap. Anche il mercato delle ipoteche beneficiò di tutte queste novità, attraverso le obbligazioni sintetiche.

L'ondata di innovazioni venne sostenuta, per tutti i primi anni Ottanta, dal rialzo dei corsi azionari e da un clima di ottimismo e fiducia nei confronti del mercato (Carlson, 2007). Il trampolino di lancio verso la sua straordinaria espansione, fu il 1984; il mese di novembre si aprì positivamente, sull'onda di previsioni di ulteriori tagli ai tassi d'interesse: il segretario al Tesoro americano, Donald Regan aveva dichiarato che il prime rate al $12 \%$ era troppo alto, preludendo così a una politica fiscale meno restrittiva; il clima era euforico anche in vista delle elezioni per la presidenza Usa; all'indomani del voto, quando la vittoria 
di Reagan iniziò ad apparire certa, Wall Street registrò un aumento di quindici punti: la Borsa aveva scommesso sulla sua vittoria.

La Borsa americana era controllata dagli operatori professionisti, favoriti dall'appoggio delle banche e dal trading degli hedge fund, costituiti da soci investitori privati che aggiravano così i regolamenti della Security and Exchange Commission; tra questi fondi quello che ebbe maggior successo fu quello lanciato dal finanziere d'origine ungherese, George Soros. Egoista, irascibile, amante dell'ostentazione, l'operatore divenne il simbolo degli anni Ottanta, il «Padrone dell'Universo», secondo la definizione di Tom Wolfe nel Falò delle vanità (Chancellor, 2000 , p. 239). Il denaro non si muoveva più con un ritmo regolare, ma circolava con la rapidità della roulette e i suoi servitori dovevano conformarsi a questo ritmo.

Il 25 agosto 1987 l'indice Dow Jones chiuse con una crescita annuale del $43 \%$, ma nonostante questo risultato il flusso di fondi era cominciato a diminuire; vi era il timore di una ripresa inflazionistica e il dollaro stava perdendo nei confronti dello yen, facendo rientrare i capitali nipponici. Il lunedì 19 ottobre 1987 il crollo del mercato azionario internazionale incominciò ad est, con il sorgere del sole; Hong Kong, Malaysia e Singapore, seguiti da alcuni mercati europei, avevano ceduto pesantemente mentre New York dormiva. Quando aprì il mercato azionario americano crollò sotto l'enorme ondata di vendite, precipitando in un sol giorno da 2.246,73 a 1.738,74 punti e perdendo il 22,61\% del proprio valore. Si trattò del maggiore crollo registrato dal Dow Jones Industrial Average nella sua storia, imparagonabile anche a quelli segnati durante la crisi del 1929 (Carlson, 2007). Alla caduta di New York fecero eco tutte le principali Borse mondiali: tra queste, Londra perse quasi l'11\%, Zurigo scese del 10,82\%, Francoforte del 7,14\%, Milano di oltre il 6\% e Parigi del 4,65\%.

Dal punto di vista macroeconomico, il livello raggiunto dal mercato azionario in agosto era eccessivamente elevato, non tanto con riferimento alle singole società quanto rispetto al contesto del periodo. Le prime avvisaglie di flessione si erano materializzate in seguito alla diffusione dei dati sul deficit commerciale americano, cresciuto dai 14,04 miliardi di dollari di maggio ai 15,71 di giugno fino ai 16,47 di luglio. A queste notizie seguì l'aumento dei tassi d'interesse e del prime rate, che causò un deprezzamento del mercato obbligazionario. Ma per molti un colpo decisivo fu dato dalla comunicazione del deficit commerciale di agosto, sceso a 15,6 miliardi di dollari, ma nettamente superiore alle 
attese. La notizia fu diffusa il 14 ottobre: i timori riguardanti il livello del tasso d'interesse originarono tre giorni di pesanti ribassi (in cui il Dow Jones perse rispettivamente il 3,81\%, il 2,39\% e il 4,60\%) e furono il preludio al crollo finanziario.

Per quanto riguarda le conseguenze della crisi e la sua propagazione, immediatamente la Fed si rivelò preoccupata di un ulteriore crollo dovuto all'irrazionalità che dominava il mercato, e quindi agì da prestatore di ultima istanza fornendo immediatamente liquidità al mercato attraverso operazioni di mercato aperto. Contrariamente a quanto era stato fatto dopo il crollo del 1929, il suo presidente, Alan Greenspan, assicurò che un partenariato era stato sancito tra la Fed e le maggiori banche americane per garantire credito in primo luogo a esse e in seguito agli operatori, così che potessero riprendere l'attività nei mercati. Nel lungo termine, non si verificò una crisi economica, sia per la tempestiva risposta della Fed; sia perché molte società quotate approfittarono della situazione per riacquistare proprie azioni a un prezzo inferiore, dando un segnale di forza; sia perché la situazione macroeconomica rimase stabile, l'occupazione crebbe e il deficit commerciale diminuì; sia perché i settori di beni e servizi non collegati al mercato azionario non subirono gli effetti della crisi, mostrando quanto fosse in salute il nucleo di imprese non quotate (Carlson, 2007).

Per la prima volta, nella storia delle crisi finanziarie, appariva però a tutti evidente che le ragioni psicologiche avevano avuto una parte dominante. Si trattava di un caso d'irrazionalità endemica che aveva portato al crearsi di una bolla speculativa, un'anormale espansione di situazioni positive la cui crescita aveva indotto l'aumento dei prezzi delle azioni. Il miraggio di facili e immediati guadagni era stato irresistibile e aveva attratto la massa investitrice, con un richiamo tale da far dimenticare ogni invito alla prudenza o a comportamenti responsabili. Robert J. Shiller condusse un'analisi sull'aspetto psicologico delle scelte in questo specifico evento finanziario, esaminando un campione di 900 investitori privati e istituzionali e chiedendogli quale fosse stato il fattore maggiormente responsabile del crack: i fondamentali economici, come i profitti societari o i tassi d'interesse, o la psicologia dell'investitore. I due terzi degli intervistati incolpò la psicologia dell'investitore più che dare una giustificazione economica, mentre il $24 \%$ disse che il crollo era stato causato da una sorta di comportamento irrazionale da parte degli sottoscrittori dei titoli. Shiller concluse che «i suggerimenti che abbiamo ottenuto sulle cause che crack sono che la gente reagisce 
reciprocamente con pronunciata attenzione ed emozione». Per gli investitori l'informazione più rilevante fu il prezzo in picchiata dei prezzi, non un cambiamento fondamentale nella valutazione futura degli utili societari. Come il prezzo delle azioni crollò, gli investitori adottarono la «mentalità della folla», portando alle vendite a cascata (Shiller, 2000, pp. 177-189).

Anche nel nostro paese, le spiegazioni in merito alla caduta degli indici azionari vennero cercate oltre i consueti strumenti economicofinanziari, nella dimensione psicologica dell'evento; il 20 ottobre 1987, Carlo Bastasin scriveva su "Il Sole 24 Ore" che «il popolo della Borsa si è riempito di domande senza risposta sul calo degli indici delle Borse mondiali. L'incapacità stessa di trovare una spiegazione era di fatto la risposta ... Ed il panico di ieri è frutto della tensione a cui sono sottoposti, da qualche mese, gli spiriti animali degli investitori internazionali». Con maggiore forza Adamo Gentile sottolineava che «è molto probabile che per esaminare l'attuale momento delle Borse mondiali più che un analista ci voglia uno psicologo. Infatti in una giornata come quella di ieri non è difficile capire che la componente emotiva dei vari mercati ha preso il sopravvento sulla logica finanziaria» (20 ottobre 1984, Il Sole 24 Ore).

\section{Considerazioni CONCLUSIVE}

Le storie che abbiamo appena raccontato confermano che, forse, a nessun altro fenomeno storico economico quanto alle crisi finanziarie si attagli altrettanto bene l'incipit dell'Anna Karenina di Tolstoj: «tutte le famiglie felici si somigliano; ogni famiglia infelice è invece disgraziata a modo suo» (citato in Conti, 2006). Differiscono la natura del mutamento, l'oggetto della speculazione, le forme dell'espansione del credito e l'episodio che provoca il rivolgimento; mutano profondamente i contesti economici e culturali, i modi di produzione, la sofisticazione della finanza, i modelli di consumo privato e gli spazi dell'intervento pubblico; tra l'economia olandese del Seicento e quella dell'epoca di Reagan c'è una differenza abissale, quasi si trattasse di due mondi diversi.

Eppure si tratta di differenze che riguardano più la morfologia delle singole crisi e meno la natura delle loro cause. L'entropia segna implacabilmente la direzione del tempo e ogni evento è unico; per 
capirle non si può prescindere dall'ambito della microstoria combinato con l'analisi dei fenomeni di psicologia individuale e collettiva, di razionalità circoscritta e di irrazionalità globale e contagiosa. Ma, come ha sostenuto in proposito Charles Kindleberger, uno dei più influenti studiosi della storia delle crisi finanziarie, «se si può prendere a prestito un proverbio francese, più qualcosa cambia, più rimane la stessa. I dettagli proliferano, le strutture permangono» (Kindleberger, 1989, p. 24). Sulla base di questa ipotesi, sono state costruite diverse interpretazioni riconducibili a due approcci prevalenti, il primo, che riflette l'impostazione monetaristica di Milton Friedman, Ann Schwartz e Phillip Cagan, per i quali la trasmissione delle crisi finanziarie si connette al meccanismo dei pagamenti internazionali, il secondo che si identifica con l'apporto critico di Hyman Minsky e dello stesso Kindleberger, si richiama alla tradizione di pensiero keynesiana, e insieme, fisheriana (Pecorari, 2006); entrambe le chiavi interpretative esplicitano il ruolo di fattori non tradizionalmente economici, ma è il secondo modello a mettere al centro della meccanica delle crisi finanziarie - ad individuare una parte fondamentale della loro intima essenza - nel comportamento irrazionale degli attori, nelle reazioni maniacali tanto dei protagonisti come delle vittime.

Secondo questa lettura, ad un certo punto interviene un fattore che genera uno spostamento (displacement) delle prospettive economiche dando il via a nuove occasioni di investimento e di guadagno, alimentate dall'aumento del credito (i tulipani nel 1634-37, le azioni delle compagnie commerciali nel 1720 , quelle delle società ferroviarie nel 1844-47, il mercato finanziario nel 1929 e nel 1987); a questo segue un effetto secondario positivo, poiché la crescita del prezzo delle azioni del nuovo fattore induce investitori inesperti ad affacciarsi al mercato azionario, determinando però un'euforia che segnala il primo indebolimento della razionalità degli investitori. Ci si getta nelle nuove opportunità in modo quasi maniacale; la speculazione quindi si allarga a nuovi prodotti finanziari e si collocano nuove società senza una base operativa, ma solo per sfruttare l'euforia; il surriscaldamento deriva dall'esiguità del liquido necessario sia rispetto al prezzo dei beni sia rispetto alle possibili variazioni di prezzo; si concede infatti di acquistare sia a premio o a rate, mentre poi è possibile vendere l'obbligazione in anticipo e incassare subito il guadagno. Le imprese e le famiglie appena vedono che altri fanno profitti con acquisti e rivendite speculative, tendono ad accodarsi: «Monkey see, monkey do»; agisce l'istinto 
del comportamento gregario; nulla disturba così tanto il nostro benessere e il nostro buon senso quanto vedere un amico diventare ricco (Kindleberger, 1989, p. 19). Vengono coinvolti settori della popolazione che di solito si astengono da queste avventure; la speculazione in cerca di rapidi profitti allontana dal comportamento razionale, dando il via a quella che viene indicata come 'manìa' (espressione che accentua l'irrazionalità) $)^{2}$ o 'bolla' (termine che fa presagire lo scoppio). Quindi si diffondono il raggiro e la frode, preludio della crisi che arriva quando le aspettative si rovesciano, o perché i prezzi smettono di crescere o per qualunque altro elemento che segnali l'eccesso di speculazione. Se non interviene nessun agente esterno - il prestatore di ultima istanza, ruolo che le banche centrali iniziano a svolgere dal 1866 a bloccare il panico e a rovesciare le aspettative, il crack finanziario raggiunge sì un punto di svolta inferiore, ma questo può essere così basso, come nel caso della Francia di Law, o del 1929, da provocare il fallimento di numerose banche e imprese e da ridurre in miseria larga parte della popolazione.

In tempi più recenti, alcuni economisti, studiosi di finanza comportamentale, ${ }^{3}$ hanno approfondito le basi psicologiche del mercato finanziario, proponendo una serie di categorie in cui collocare gli atteggiamenti non riconducibili alla razionalità; pur con tutti i distinguo del caso, si tratta di modelli che possono aiutare lo storico economico a inquadrare meglio alcune reazioni del passato: al pensiero magico, che è responsabile di un'eccessiva fiducia per quanto riguarda i meccanismi speculativi pur non avendone una spiegazione razionale, si possono così ricondurre i comportamenti di quelli che investirono nella Compagnie des Indies e in quella dei Mari del Sud senza nessuna idea del meccanismo su cui poggiava artificialmente il continuo rialzo; all'euristica della rappresentatività, in base alla quale si tende ad esprimere giudizi, ipotizzando che i modelli futuri assomiglieranno a quelli passati, senza considerare sufficientemente la probabilità che questo si ripeta, appartengono i giudizi di economisti, quali Irving Fischer, e di molta pubbli-

2 Nella sua quarta edizione del volume Manias, Panics and Crashes C. Kindlebergerer (2000) ha contato dal 1637 ad oggi almeno 50 «world class manias».

3 Robert J. Shiller, autore di Euforia irrazionale. Analisi dei boom di Borsa (Bologna, Il Mulino, 2000) ha confessato di avere scritto questo libro quasi a quattro mani, con la supervisione e l'aiuto della moglie, psicologa clinica. 
cistica, che alla vigilia della crisi del 1929 profetizzavano un'ulteriore crescita del Dow Jones (Shiller, 2000, pp. 202-3); dalla paura a non conformarsi derivava poi il timore dell'economista di Princeton, che, pochi mesi prima del crollo di Wall Street, affermava che «Il consenso del giudizio di milioni di investitori, in quell'ammirevole mercato ... è che $\mathrm{i}$ titoli azionari non sono sopravvalutati» (citato in Galimberti, 2002, p. 22).

La conoscenza di queste storie e la consapevolezza delle relative dinamiche comportamentali sono utili per superare l'assunto di razionalità del mercato e per rendersi conto della complessità dei fenomeni finanziari, che richiedono, per essere intimamente compresi, un approccio interdisciplinare. Le crisi finanziarie hanno da sempre accompagnato il capitalismo, che ha cercato di tenerle sotto controllo senza mai riuscire ad eliminarle, perché i sistemi sono in continua mutazione e le leggi vigenti diventano obsolete. Le crisi sono molto spesso dei punti di svolta, dei crinali, (come ricorda il loro etimo, dal greco krinein separare), in cui il paradigma economico si assesta su nuovi binari e verso nuove direzioni. In una società libera non possiamo proteggere completamente le persone senza negare loro la possibilità di realizzarsi come vogliono; ma se è vero che non è possibile impedire ad ognuno to make a foolish of himself, è altrettanto vero che è possibile impedire ai più furbi di ingannare $\mathrm{i}$ meno informati e i meno consapevoli degli effettivi meccanismi delle attività finanziarie, da quelli macroeconomici a quelli decisionali.

\section{BIBLIOGRAFIA}

G.A. Akerlof, R.J. Shiller, Animal spirits. How Human Psychology Drives the Economy, and Why It Matters for Global Capitalism, Princeton and Oxford, Princeton University Press, 2009.

C. Bastasin, "Mesi di dubbio partoriscono il grande crollo", in "Il Sole 24 Ore" del 20 ottobre 1987.

M. Carlson, "A Brief History of the 1987 Stock Market Crash with a Discussion of the Federal Reserve Response", in "Finance and Economics Discussion Series”, n. 13, 2007.

E. Chancellor, Un mondo di bolle. La speculazione finanziaria dalle origini alla "new economy", Roma, Carocci, 2000.

G. Conti, "Il crac del 1873", in P. Pecorari (a cura di), Crisi e scandali bancari nella Storia d'Italia, Venezia, Istituto Veneto di Scienze, Lettere ed Arti, 2006, pp. 9-28. 
M. Fornasari, Finanza d'impresa e sistemi finanziari. Un profilo storico, Torino, Giappichelli, 2006.

P.M. Garber, "Who put the mania in the tulipmania?" in E.N. White (ed), Crashes and Panics. The lessons from history, Homewood, New York University, 1990.

F. Galimberti, Economia e pazzia. Crisi finanziarie di ieri e di oggi, Bari, Laterza, 2008.

A. Gentile, "Quando è la paura a sconvolgere la logica economica e finanziaria", in "Il Sole 24 Ore" del 20 ottobre 1987.

J. Gleeson, L'uomo che inventò il denaro. La storia vera di John Law, libertino, giocatore d'azzardo, assassino e padre dell'economia moderna, Milano, Rizzoli, 2000.

C.P. Kindleberger, Manias, panics, and crashes. A history of financial crises, London, Macmillan, 1989.

C.P. Kindleberger, Storia delle crisi finanziarie, Bari, Laterza, 1991.

C. Mackay, La pazzia delle folle ovvero le grandi illusioni collettive, Milano, Il Sole 24 Ore, 2000.

A.E. Murphy, John Law. Economic Theorist and Policy-Maker, Oxford, Oxford University Press, 1997.

P. Pecorari, "Le crisi bancarie e finanziarie: un problema storiografico aperto. Introduzione al convegno", in P. Pecorari (a cura di), Crisi e scandali bancari nella Storia d'Italia, Venezia, Istituto Veneto di Scienze, Lettere ed Arti, 2006.

L.D. Neal, "How the South Sea bubble was blown up and burst: a new look at old data", in E.N. White (ed), Crashes and Panics. The lessons from history, Homewood, New York University, 1990.

R.J. Shiller, Euforia irrazionale. Analisi dei boom di Borsa, Bologna, Il Mulino, 2000.

J. de la Vega, Confusion de Confusiones, Amsterdam, 1688 in appendice a Mackay, 2000.

E.N. White, "When the ticker ran late: the stock market boom and crash of 1929", in E.N. White (ed), Crashes and Panics. The lessons from history, Homewood, New York University, 1990. 